\title{
Effects of Directivity on Wireless Network Complexity
}

\author{
Arta Cika and Justin P. Coon \\ Department of Engineering Science \\ University of Oxford \\ Parks Road, Oxford, UK, OX1 3PJ \\ Email: \{arta.cika and justin.coon $\} @$ eng.ox.ac.uk
}

\author{
Sunwoo Kim \\ Department of Electronic Engineering \\ Hanyang University \\ South Korea \\ Email: remero@hanyang.ac.kr
}

\begin{abstract}
We study the effect of anisotropic radiation on wireless network complexity. To this end, we model a wireless network as a random geometric graph where nodes have random antenna orientations as well as random positions, and communication is affected by Rayleigh fading. Complexity is quantified by computing the Shannon entropy of the underlying graph model. We use this formalism to develop analytic scaling results that describe how complexity can be controlled by varying key system parameters such as the transmit power and the directivity of transmissions in large-scale networks. Our results point to striking contrasts between power scaling and directivity scaling in the large connection range regime.
\end{abstract}

Index Terms-Graph entropy, random geometric graphs, directivity, network topology.

\section{INTRODUCTION}

In an age of shrinking cell sizes, growing connection densities, machine-type applications and the Internet of Things, network complexity is becoming a topic of increasing interest. A fundamental understanding of complexity in this context can help engineers to predict network performance, network dynamics and devise management and transmission protocols that are scalable to larger systems. Of course, complexity can mean many things. In this contribution, we study network complexity by focusing on the topology of a wireless network. We begin from the perspective that node positions and prevailing channel conditions are unknown a priori, which inherently induces randomness in the network topology. Hence, our focus in this paper is on characterizing the uncertainty of this topology under certain assumptions on the underlying statistical processes that model the node locations and fading conditions. This uncertainty inherently relates to the complexity of the network.

Topological uncertainty has been studied in a number of scientific fields for many years through the mathematical formalism of graph entropy [1]. Fascinating correspondences have been drawn between entropy maximising edge distributions and quantum mechanical systems [2]. Many different notions of graph entropy have been developed under the collective aim of better understanding properties of complex networks [3]. Until recently, however, research has been focused on non-spatial networks, that is to say networks in which the likelihood of a connection between two nodes existing is not a function of the distance between them (e.g., Erdős-Rényi (ER) graphs). Yet, it is increasingly becoming important to recognize the spatial dependences in networks, and wireless systems exhibit perhaps the most well understood spatial characteristics of engineered systems today. About ten years ago, a couple of early studies of network organization identified the importance of topological uncertainty [4], [5], but the frameworks that were employed in those investigations did not lend themselves to quantitative analysis. Very recently, two analytic studies were published that give the first accounts of the scaling properties of topological uncertainty in wireless ad hoc networks [6], [7]. Apart from these examples, this topic has been left relatively unexplored.

From a practical perspective, the availability of topology information at each node is critical [8]. This information provides a view of connectivity at a fundamental level, but it can also be exploited in practice to choose modulation, coding, and routing protocols [9], [10]. Indeed, in topologybased routing protocols for mobile ad hoc networks, nodes collect topology information to make routing decisions [11].

In this contribution, we extend the work reported in [6], [7] to systems that employ directive transmissions. Our study is motivated by the increasing focus on the use of millimeter wave bands in future wireless systems [12]. We model a wireless network as a random geometric graph (RGG) and quantify the network complexity by studying the Shannon entropy of the underlying graph model. In our network model, nodes have random antenna orientations as well as random spatial positions, and communication links are affected by Rayleigh fading. We examine the behavior of the RGG entropy as the number of nodes $n$ in the network grows large while the typical connection range and directivity vary monotonically with $n$. Our analysis is conducted for two different antenna radiation patterns: a cardioid and a sectorized pattern. The main contributions of this paper are the development of four lemmas that quantify various scaling conditions that must be met for the network entropy to tend to a finite, positive limit. These results help us to understand how system parameters such as transmit power and antenna gain affect complexity in largescale networks.

The structure of the paper is as follows. In section II, we explain the details of the network, the pairwise connection 
model, and the antenna gain model considered in this work. We quantify complexity for wireless networks in section III by analyzing the Shannon entropy of RGGs considering two different radiation patterns. In sections IV and $\mathrm{V}$, we present the aforementioned scaling lemmas. Finally, in section VI, we present our conclusions.

Notation: We use $\mathbb{E}[\cdot]$ to denote the expectation operator. Given two nonnegative functions defined on some subset of the real numbers, we say that $f(x)=O(g(x))$ if and only if there exist constants $x_{1}, c_{1}>0$ such that $f(x) \leq c_{1} g(x)$ for all $x>x_{1}$. Moreover, we say that $f(x)=\Omega(g(x))$ if and only if there exist constants $x_{2}, c_{2}>0$ such that $f(x) \geq c_{2} g(x)$ for all $x>x_{2}$. The notation $f(x)=o(g(x))$ implies that $f(x) / g(x) \rightarrow 0$ in the appropriate limit. The $W_{m}(x)$ function is the lower branch $\left(-1 / e \leq x<0\right.$ and $\left.W_{m} \leq-1\right)$ of the solution to $x=W \exp W$.

\section{SYSTEM MODEL}

\section{A. Network Model}

We consider a network of $n$ nodes representing wireless devices located randomly in a two-dimensional space $\mathcal{K}_{2}$ (with volume $\mathcal{V}$ ). Each node is equipped with a directional antenna that is randomly oriented in the interval $[0,2 \pi)$. The locations of the nodes, $\mathbf{r}_{1}, \mathbf{r}_{2}, \ldots, \mathbf{r}_{n}$, are independent and identically distributed in $\mathcal{V}$ and are modelled as a binomial point process (BPP). Although some of the results disclosed below can be applied to general inhomogeneous processes, it will be assumed the BPP is homogeneous throughout this paper.

We model a wireless network as an instance of a soft undirected RGG [13]. The original hard RGG model, also called the unit disk or hard connection model, captures the distance dependence and randomness in the connectivity of the nodes in a spatial network [14]. The soft RGG model is employed here to enable statistical fading effects in the channel to be accounted for. Any two nodes are directly connected if they satisfy a specific pair connection criterion (outlined below). The set of all possible graphs formed from every combination of pairwise connections is denoted by $\mathcal{G}$, where $|\mathcal{G}|=2^{n(n-1) / 2}$.

\section{B. Point-to-Point Connections}

Two nodes, $i$ and $j$, are directly connected with probability $p\left(r_{i, j}\right):=p_{i, j}$, where $r_{i, j}=\left\|\mathbf{r}_{i}-\mathbf{r}_{j}\right\|$ is the Euclidean distance between $i$ and $j$. In our model, nodes have an orientation as well as a position and their ability to communicate depends on both of these factors. We define the connection probability between transmitting node $i$ and receiving node $j$ as the complement of the outage probability with respect to a mutual information threshold $I$. For a single-input single-output (SISO) transmission in a Rayleigh fading channel, this probability is given by

$$
p_{i, j}=\mathbb{P}\left(\log _{2}\left(1+\mathrm{SNR}_{i, j}\left|h_{i, j}\right|^{2}\right) \geq I\right)
$$

where $\mathrm{SNR}_{i, j}$ denotes the average received signal-to-noise ratio and $h_{i, j}$ is the channel transfer coefficient with $\mathbb{E}\left[|h|^{2}\right]=1$.
Assuming identical lossless antennas, we can employ the Friis transmission formula to obtain the relation

$$
\mathrm{SNR}_{i, j} \propto g_{T}\left(\phi_{i j}\right) g_{R}\left(\phi_{j i}\right) r^{-\eta}
$$

where $g_{T}$ and $g_{R}$ model the gains of the transmit and receive antennas, respectively, $r$ is the distance between nodes $i$ and $j$, and $\eta$ is the path loss exponent (typically $\eta \geq 2$ ). The arguments of the gain functions $\phi_{i j}$ and $\phi_{j i}$ signify the orientations of nodes $i$ and $j$ relative to each other. That is to say, these angles encompass the individual antenna orientations in the plane as well as the observation angles between the transmitter and the receiver. For the remainder of the paper, we assume $g_{T}=g_{R}$, and we enforce the normalization

$$
\int_{0}^{2 \pi} g(\phi) \mathrm{d} \phi=2 \pi
$$

Based on the Rayleigh fading and Friis transmission models, we see that the pair connection probability for nodes $i$ and $j$ can be succinctly written as

$$
p_{i, j}=\exp \left(\frac{-\left(r / r_{i}\right)^{\eta}}{g\left(\phi_{i j}\right) g\left(\phi_{j i}\right)}\right)
$$

where $r_{i}$ is the typical isotropic connection range. In the case of anisotropic radiation, the typical connection range $r_{0}$ can be defined as

$$
r_{0}=r_{i}\left(g\left(\phi_{i j}\right) g\left(\phi_{j i}\right)\right)^{\frac{1}{\eta}} .
$$

Note that in the limit $\eta \rightarrow \infty$, the connectivity between nodes is no longer probabilistic and we recover the popular hard connection model where connections have a fixed range equal to $r_{0}$.

\section{Antenna Gain Functions}

The gain or directivity of an antenna is the ratio of the radiation intensity in a given direction to the average of the radiation intensity over all directions [15]. Quite often, directivity and gain are used interchangeably. The difference is that directivity neglects antenna losses. Since these losses in most classes of antennas are usually quite small, the directivity and antenna gain will be considered approximately equal in this paper. Here, we examine some practical functions for $g$ that represent realistic propagation models.

1) Cardioid Pattern: The cardioid pattern is a good approximation to the wide-angle unidirectional radiation pattern exhibited by a patch antenna in a plane [15]. The $\epsilon$-cardioid gain function is defined as

$$
g(\phi)=1+\epsilon \cos \phi, \quad 0 \leq \phi \leq 2 \pi
$$

where $\epsilon \in[0,1]$ is a parameter that defines the degree of deformation with respect to the isotropic case $(\epsilon=0)$. Radiation patterns with different values of $\epsilon$ are shown in Fig. 1. 


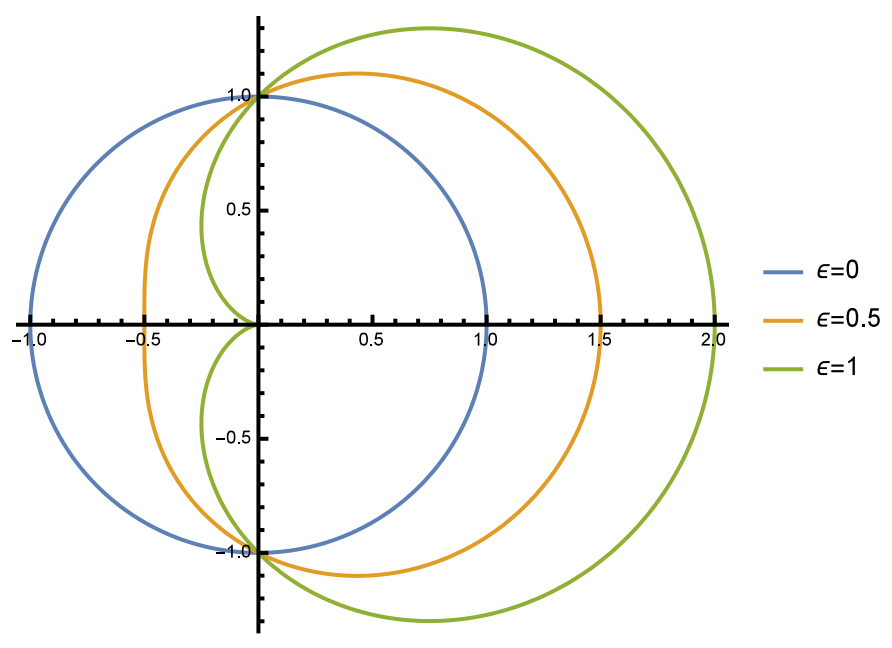

Fig. 1. Polar plot of the cardioid radiation pattern for different values of $\epsilon$.

2) Sectorized Pattern: Many practical antennas are designed to exhibit sectorized intensity patterns. For example, antenna arrays consisting of half-wave dipoles separated by half of a wavelength in one direction radiate along a main lobe with several small side lobes [15]. We can approximate the gain function for a sectorized antenna in a very general manner by defining

$$
g(\phi)= \begin{cases}\nu \lambda \cos ^{\rho}(\lambda \phi), & \frac{-\pi}{2 \lambda} \leq \phi \leq \frac{\pi}{2 \lambda} \\ 0, & \text { otherwise }\end{cases}
$$

where $\lambda \geq 1$ defines the directivity of the beam and $\rho \in(0,1)$ signifies the degree of sectorization. The normalization constant is

$$
\nu=\frac{2 \sqrt{\pi} \Gamma\left(1+\frac{\rho}{2}\right)}{\Gamma\left(\frac{1+\rho}{2}\right)} .
$$

Sectorized antennas are used extensively to increase the capacity of wireless systems as they are ideal for covering specific areas over a prescribed angle. If $\rho \rightarrow 1$, in (7) we recover the gain function of an end-fire array, a very high directional antenna ideal for point-to-point communications [15]. To better understand the roles of parameters $\{\rho, \lambda\}$, in Fig. 2 we have plotted the gain function of a sectorized antenna for different values of these parameters. A large $\lambda$ produces a highly directional gain function, and $\rho \simeq 0$ yields an isotropic sectorized pattern with larger $\rho$ giving a smoother function. From these observations, we expect that $\lambda$ will be the main parameter affecting topological uncertainty in our network model.

\section{Network COMPleXity (GRAPH ENTROPY)}

Uncertainty is naturally quantified using the notion of entropy. To understand the complexity of networks, a different number of entropy measures have been introduced [16]-[19]. We quantify the complexity of a wireless network by quantifying the Shannon entropy of the underlying RGG ensemble,

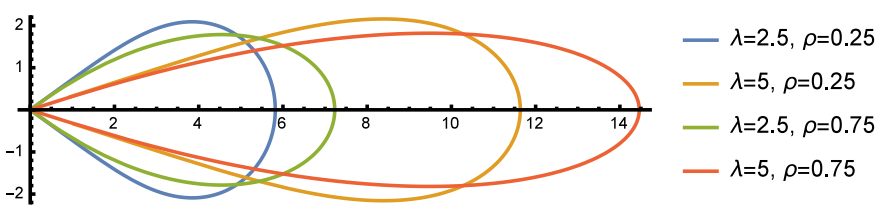

Fig. 2. Polar plot of the sectorized radiation pattern for different values of $\lambda$ and $\rho$.

which is defined as the logarithm of the number of typical networks in the ensemble. The entropy of a graph $G$ can be written as

$$
H(G)=\mathbb{E}\left[-\log _{2} \mathbb{P}(G)\right]=-\sum_{G \in \mathcal{G}} \mathbb{P}(G) \log _{2} \mathbb{P}(G) .
$$

The following upper bound on $H(G)$ was derived in [6]:

$$
h(n):=\left(\begin{array}{l}
n \\
2
\end{array}\right) H_{2}(\bar{p}) \geq H(G)
$$

where

$$
H_{2}(\bar{p})=-\bar{p} \log _{2} \bar{p}-(1-\bar{p}) \log _{2}(1-\bar{p})
$$

is the binary entropy function and $\bar{p}$ is the average pairwise connection probability. In [6], $\bar{p}$ was obtained by averaging the pair connection function over the pair distance distribution. Here, we must also average over the transmitter and receiver orientations. This effectively manifests as an independent averaging over the angles $\phi_{i j}$ and $\phi_{j i}$ :

$$
\bar{p}=\iiint p_{i, j} f_{r}(r) f_{i j}\left(\phi_{i j}\right) f_{j i}\left(\phi_{j i}\right) \mathrm{d} r \mathrm{~d} \phi_{i j} \mathrm{~d} \phi_{j i}
$$

where $f_{r}, f_{i j}$, and $f_{j i}$ are the density functions in the respective variables. This simple integral somewhat hides a subtlety that is worthy of note. Averaging over the distribution $f_{r}$ inherently accounts for pairwise rotational symmetry (i.e., orbital symmetry). As a result, the $\mathrm{d} \phi_{i j}$ and $\mathrm{d} \phi_{j i}$ integrals act to average over the transmitter and receiver orientations separately (i.e., spin averaging). Thus, (12) is a slight abuse of notation given the original definitions of the gain function arguments stated in section II-B, but mathematically it is entirely correct.

Expressions for the pair distance density $f_{r}$ exist for simple bounding geometries and homogeneous node placement. The interested reader is referred to [20] for further information. For now, we refrain from defining a specific geometry, and in fact most of the ensuing analysis will apply for fairly general geometries. Regarding the orientation distributions, we assume that the relative orientations are uniform in an interval. Hence, the orientation density functions, which we assume to be equal (i.e., $f_{i j}=f_{j i}$ ), are given by

$$
f_{i j}(\phi)= \begin{cases}\frac{1}{\Delta}, & \frac{-\Delta}{2} \leq \phi \leq \frac{\Delta}{2} \\ 0, & \text { otherwise. }\end{cases}
$$

For the cardioid pattern, $\Delta=2 \pi$. For the sectorized pattern, $\Delta=\pi / \lambda$. Note that this implies the model considered for the sectorized pattern is one where nodes perform beam steering 


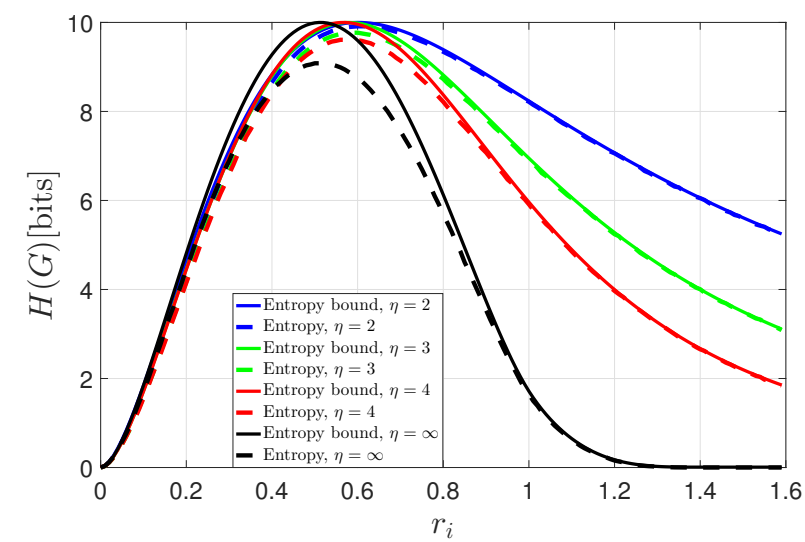

Fig. 3. Entropy of an RGG with $n=5$ nodes with isotropic radiation. Solid lines: upper bound $h(n)$. Dashed lines: numerical simulations for $H(G)$.

to a coarse degree when attempting to communicate. That is, nodes vaguely orient their transmissions toward each other, but the exact orientation is still random. This is a very practical model for, e.g., future millimeter wave networks.

\section{A. Numerical Results}

The standard case of isotropic radiation was studied in [6]. In that work, scaling laws were derived that link the typical isotropic connection range $r_{i}$ and the number of nodes $n$ to the entropy bound $h(n)$. The results of [6] are reproduced in Fig. 3 for convenience and to provide a benchmark for the anisotropic analysis detailed below.

In Figs. 4 and 5, we begin to explore the effect that anisotropic radiation has on entropy. Here, we plot the RGG entropy $H(G)$ and the entropy bound $h(n)$ against the typical isotropic connection range $r_{i}$ for different path loss exponents $\eta=2,3,4, \infty$. We consider a network with $n=5$ nodes confined within a unit square in $\mathbb{R}^{2}$. To produce these figures, $\bar{p}$ in (12) is obtained by averaging over all possible orientations as well as the pair distance. These results offer some interesting observations. The first point to note is that the bound is relatively tight, particularly for soft connection functions (finite path loss values). Furthermore, we see that the uncertainty in the network topology decreases as $r_{i} \rightarrow 0$ and $r_{i} \rightarrow \infty$. Finally, the rate of decay of entropy is higher for an antenna with a more directional beam (Fig. 5), compared to an antenna with a more isotropic radiation pattern (Fig. 4).

\section{B. A Note on Entropy Growth}

Analytically, we can deduce from (10) that the bound grows like $O\left(n^{2}\right)$ if all other system parameters are held constant. This agrees with intuition since the addition of a node to the network increases the number of fading links (by one less than the total number of nodes), which will in turn increase the uncertainty in the network topology. From a fundamental perspective, unbounded network complexity may be undesirable. To lend an operational interpretation for wireless networks, consider the unchecked evolution of the Internet of Things, where mesh

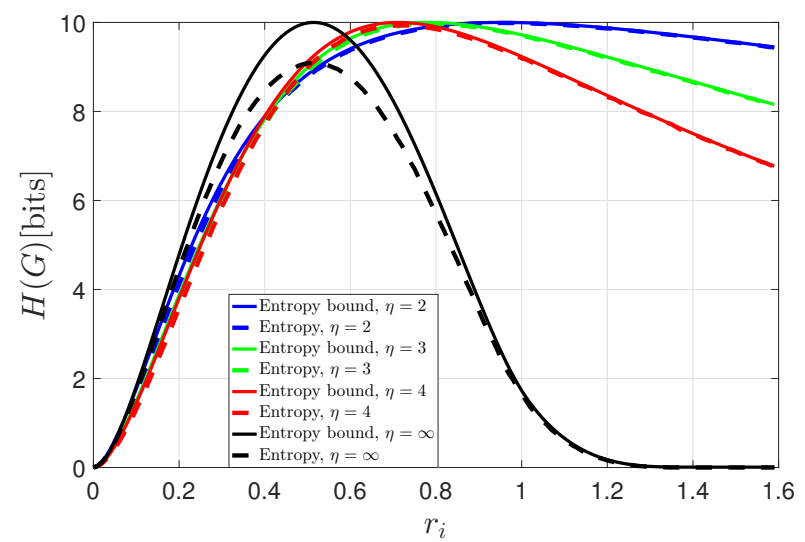

Fig. 4. Entropy of an RGG with $n=5$ nodes and cardioid radiation patterns. $H(G)$ is expressed in bits. The directivity parameter is $\epsilon=1$. Solid lines: upper bound $h(n)$. Dashed lines: numerical simulations for $H(G)$.

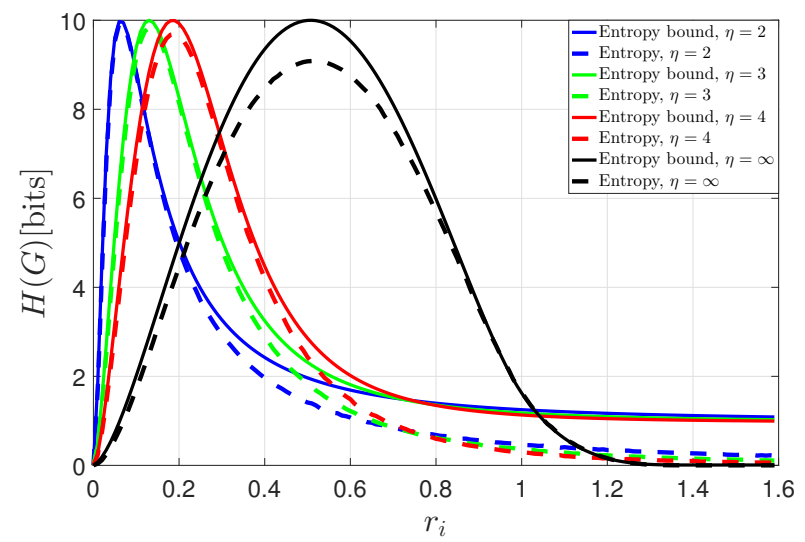

Fig. 5. Entropy of an RGG with $n=5$ nodes equipped with sectorized antennas. $H(G)$ is expressed in bits. The directivity parameter is $\lambda=5$ and the degree of sectorization is $\rho=0.5$. Solid lines: upper bound $h(n)$. Dashed lines: numerical simulations for $H(G)$.

topologies are employed for reliability and coverage purposes. With node densities set to exceed one connection per square meter in the not-too-distant future, it is easy to imagine the case where complexity even on a local scale becomes unmanageable. In this context, the entropy of the network ensemble would define the number of bits needed to store the network topology (or a portion thereof) or indeed to convey this information to other nodes in the network. Without mitigating entropy growth in such evolving networks, huge operational problems would soon be encountered. This postulate motivates our investigation of the scaling behavior of entropy with respect to system properties such as the typical connection range and directivity.

\section{Small Typical Isotropic Connection Range}

The typical isotropic connection range $r_{i}$ depends on the signal power, the transmission wavelength and the power of the long-time average background noise at the receiver. For instance, if we employ a simplified path loss model [21], it is 
straightforward to show that $r_{i} \propto P_{T}^{1 / \eta}$. Thus, $r_{i}$ is a crucial engineering parameter that can be used to control network entropy.

Our goal here is to develop scaling laws that link $r_{i}$, the directivity parameters $\{\epsilon, \lambda\}$, and the number of nodes $n$ to the entropy bound $h(n)$ as $r_{i} \rightarrow 0$. In this scenario entropy tends to zero, which in the limit corresponds to the case of a completely disconnected network. Hence, it is of fundamental interest to ascertain the conditions under which such a scenario would (or would not) occur.

To make progress, we must calculate $\bar{p}$ in (12), which is done by first integrating over the pair distance $r$ and subsequently the angles $\phi_{i j}$ and $\phi_{j i}$. As mentioned above, particular bounding regions and BPP intensities give rise to specific expressions for $f_{r}$. However, if $\mathcal{K}_{2}$ is a compact, convex set and the nodes are distributed homogeneously in this region, $f_{r}$ has the following simple, general form for small $r$ [6]:

$$
f_{r}(r) \simeq \frac{2 \pi r}{a}\left(1-\frac{l r}{\pi a}\right)
$$

where $a$ and $l$ are the area and perimeter length of $\mathcal{K}_{2}$. Note that for a domain with diameter $D$, we would perform the average over the pair distance variable by integrating over the finite interval $[0, D]$. However, the exponential decay of $p_{i, j}$ coupled with the small $r_{i}$ assumption allows us to expand this interval to $[0, \infty)$, which leads to a closed form result without significantly sacrificing accuracy [6]. Finally, noting that $f_{i j}=f_{j i}=1 / \Delta$, we average over the transmitter and receiver orientation angles to obtain

$$
\begin{aligned}
\bar{p}=\frac{1}{\Delta^{2}} \frac{2 \pi r_{i}^{2}}{a \eta} \Gamma\left(\frac{2}{\eta}\right)\left(S_{2 / \eta}[g]\right)^{2} & \\
& -\frac{1}{\Delta^{2}} \frac{2 l r_{i}^{3}}{a^{2} \eta} \Gamma\left(\frac{3}{\eta}\right)\left(S_{3 / \eta}[g]\right)^{2}
\end{aligned}
$$

where

$$
S_{y}[g]=\int_{0}^{2 \pi} g(\phi)^{y} \mathrm{~d} \phi .
$$

Below, we will exploit (15) and (16) to evaluate the entropy bound (10). We will then derive two lemmas that give conditions on $r_{i}$ and the directivity parameters $\{\epsilon, \lambda\}$ that must be satisfied in order to ensure the graph is not completely disconnected.

\section{A. Cardioid Pattern}

For a cardioid pattern, $g$ is given by (6). Hence, we have

$$
\begin{aligned}
S_{y}[g]=\pi(1-\epsilon){ }_{2}^{y} F_{1}\left(\frac{1}{2},-y ; 1 ;-\frac{2 \epsilon}{1-\epsilon}\right) \\
+\pi(1+\epsilon)^{y}{ }_{2} F_{1}\left(\frac{1}{2},-y ; 1 ; \frac{2 \epsilon}{1+\epsilon}\right)
\end{aligned}
$$

where ${ }_{2} F_{1}\left(a_{1}, a_{2} ; b ; x\right)$ is the Gauss hypergeometric function. Combining (15) and (17) and considering the case when $r_{i} \simeq 0$, we can write the binary entropy as

$$
H_{2}(\bar{p})=\frac{u r_{i}^{2}}{\ln 2}\left(2 \ln \left(\frac{1}{r_{i}}\right)+1-\ln u\right)+O\left(r_{i}^{3} \ln r_{i}\right)
$$

where

$$
u=\frac{\Gamma(2 / \eta)\left(S_{2 / \eta}[g]\right)^{2}}{2 \pi a \eta}
$$

can be interpreted as the fractional area of a soft $\epsilon$-cardioid. Note that for any $\eta>2, S_{2 / \eta}[g]$ is monotonically decreasing in $\epsilon$ and thus so is the entropy bound. Hence, for finite $n$, network complexity can be controlled somewhat by varying directivity.

In the large $n$ limit, we can exploit (18) to develop the following lemma that elucidates the conditions under which the entropy bound tends to a positive limit as $r_{i} \rightarrow 0$.

Lemma 1: As $n \rightarrow \infty$, the entropy of a network of nodes with cardioid radiation patterns in $\mathcal{K}_{2}$ can be bounded away from zero only if

$$
r_{i}^{2} \ln \left(\frac{1}{r_{i}}\right)=\Omega\left(\frac{1}{n^{2}}\right) .
$$

The entropy bound will tend to a limit $h(n) \rightarrow b_{h}>0$ as $n \rightarrow \infty$ if

$$
r_{i}(n)=\exp \left(\frac{1}{2} W_{m}\left(-\frac{2 b_{h} \ln 2}{n(n-1) u}\right)\right) .
$$

Proof: See [6].

Lemma 1 provides interesting insight into the complexity of networks with nodes that exhibit cardioid-like connection ranges spatially. It not only describes the scaling behavior of the typical isotropic connection range $r_{i}$ but also that of the transmit power. Indeed, to achieve stability in entropy in limit of large $n$, the transmit power must scale like

$$
P_{T} \propto\left(n^{2} \ln n\right)^{-\frac{\eta}{2}} \text {. }
$$

Also note that the scaling result given above depends upon the directivity parameter $\epsilon$ through $u$.

\section{B. Sectorized Pattern}

For a sectorized radiation pattern, the typical connection range $r_{0}$ is given by

$$
r_{0}=\tilde{r} \cos ^{\frac{\rho}{\eta}}\left(\lambda \phi_{i j}\right) \cos ^{\frac{\rho}{\eta}}\left(\lambda \phi_{j i}\right)
$$

where $\tilde{r}=r_{i}(\nu \lambda)^{\frac{2}{\eta}}=\max _{\phi_{i j}, \phi_{j i}}\left\{r_{0}\right\}$. In this case, we have

$$
S_{y}[g]=\frac{\sqrt{\pi} \nu^{y} \lambda^{y-1} \Gamma\left(\frac{1+\rho y}{2}\right)}{\Gamma\left(1+\frac{\rho y}{2}\right)} .
$$

Combining (15) and (24) and considering the case when $\tilde{r} \simeq 0$, we can write the binary entropy as

$$
H_{2}(\bar{p})=\frac{\tilde{u} \tilde{r}^{2}}{\ln 2}\left(2 \ln \left(\frac{1}{\tilde{r}}\right)+1-\ln (\tilde{u})\right)+O\left(\tilde{r}^{3} \ln \tilde{r}\right)
$$

where

$$
\tilde{u}=\frac{2 \Gamma\left(\frac{1}{2}+\frac{\rho}{\eta}\right)^{2} \Gamma\left(\frac{2}{\eta}\right)}{a \eta \Gamma\left(1+\frac{\rho}{\eta}\right)^{2}}
$$

such that $\tilde{u} \nu^{\frac{4}{\eta}} \lambda^{\frac{4}{\eta}}-2 / 4$ is the fractional area of a soft sectorized lobe. 
Taking a similar approach as above, we wish to explore the scaling properties of entropy when $n \rightarrow \infty$ and $\tilde{r} \rightarrow 0$. In contrast to the case of the cardioid, directivity is not limited (theoretically) for the sectorized pattern. Indeed, $\lambda$ can grow without bound. However, we consider fixed $\lambda<\infty$ for this analysis. Expanding (25) near $\tilde{r}=0$ leads to the following result.

Lemma 2: As $n \rightarrow \infty$, the entropy of a network of nodes with sectorized radiation patterns in $\mathcal{K}_{2}$ can be bounded away from zero only if

$$
\tilde{r}^{2} \ln \frac{1}{\tilde{r}}=\Omega\left(\frac{1}{n^{2}}\right) .
$$

The entropy bound will tend to a limit $h(n) \rightarrow b_{h}>0$ as $n \rightarrow \infty$ if

$$
\tilde{r}(n)=\exp \left(\frac{1}{2} W_{m}\left(-\frac{2 b_{h} \ln 2}{n(n-1) \tilde{u}}\right)\right) .
$$

The form of this lemma is similar to previously reported results by the authors. Nevertheless, it possesses an important subtlety. Specifically, scaling of the product $r_{i} \lambda^{2 / \eta}$ is required. Hence, for very directive transmissions, the typical isotropic connection range must satisfy $r_{i}=o\left(\lambda^{-2 / \eta}\right)$, or equivalently $P_{T}=o\left(\lambda^{-2}\right)$, which provides some understanding of how directivity and connection range (power) can be traded off to control network complexity.

\section{LARGE TyPICAL ISOTROPIC CONNECTION RANGE}

We now turn our attention to the case where the typical isotropic connection range is large with respect to the domain of the bounding region, i.e., $r_{i} \gg D$. In this scenario, we can expand the exponential in the integrand of (12) to obtain scaling results.

\section{A. Cardioid Pattern}

First, let us focus on the cardioid pattern. Performing the required integral (i.e., (12)) yields

$$
\bar{p}=1-\frac{\mu_{\eta}}{r_{i}^{\eta}\left(1-\epsilon^{2}\right)}+O\left(\frac{1}{r_{i}^{2 \eta}}\right)
$$

where $\mu_{m}=\mathbb{E}\left[r^{m}\right]$. Using this result to calculate the binary entropy and extracting the leading term for $r_{i} \rightarrow \infty$ leads to

$$
H_{2}(\bar{p})=\frac{\eta \mu_{\eta} \ln r_{i}}{r_{i}^{\eta}\left(1-\epsilon^{2}\right) \ln 2}+O\left(\frac{1}{r_{i}^{\eta}}\right) .
$$

This analysis uncovers the following scaling result.

Lemma 3: As $n \rightarrow \infty$, the entropy of a network of nodes with cardioid radiation patterns in $\mathcal{K}_{2}$ is bounded only if

$$
\frac{r_{i}^{\eta}}{\ln r_{i}}=\Omega\left(n^{2}\right)
$$

The entropy bound will tend to a limit $h(n) \rightarrow b_{h}>0$ as $n \rightarrow \infty$ if

$$
r_{i}(n)=\exp \left(-\frac{1}{\eta} W_{m}\left(-\frac{2\left(1-\epsilon^{2}\right) b_{h} \ln 2}{\mu_{\eta} n(n-1)}\right)\right) .
$$

This lemma provides a concrete quantitative expression that we can study to observe the divergent behavior of entropy in the large $r_{i}$ and $n$ limits. The $W_{m}$ function diverges (in the negative direction) as the argument tends to zero from the left. Hence, if the radiation pattern contains a null $(\epsilon=1)$, we see that $r_{i}$ must be infinite for any finite $n$. Intuitively, we can reconcile this behavior by recognizing that the null in the beam pattern will always give rise to uncertainty in pairwise interactions, regardless of the transmit power. Practically, this means systems that employ such radiation patterns will yield high network complexity for large transmit powers.

\section{B. Sectorized Pattern}

We conclude with the large connectivity analysis for sectorized patterns. For large $r_{i}$, the average pair connection function evaluates to

$$
\bar{p}=1-\frac{\mu_{\eta} \Gamma\left(\frac{1-\rho}{2}\right)^{2}}{r_{i}^{\eta} \pi \nu^{2} \lambda^{2} \Gamma\left(1-\frac{\rho}{2}\right)^{2}}+O\left(\frac{1}{r_{i}^{2 \eta}}\right) .
$$

The entropy can be expanded in $r_{i}$ to give

$$
H_{2}(\bar{p})=\frac{\eta \mu_{\eta} \Gamma\left(\frac{1-\rho}{2}\right)^{2} \ln r_{i}}{r_{i}^{\eta} \pi \nu^{2} \lambda^{2} \Gamma\left(1-\frac{\rho}{2}\right)^{2} \ln 2}+O\left(\frac{1}{r_{i}^{\eta}}\right)
$$

which leads to thefollowing lemma.

Lemma 4: As $n \rightarrow \infty$, the entropy of a network of nodes with sectorized radiation patterns in $\mathcal{K}_{2}$ is bounded only if

$$
\frac{r_{i}^{\eta}}{\ln r_{i}}=\Omega\left(n^{2}\right) \text {. }
$$

The entropy bound will tend to a limit $h(n) \rightarrow b_{h}>0$ as $n \rightarrow \infty$ if

$$
r_{i}(n)=\exp \left(-\frac{1}{\eta} W_{m}\left(-\frac{2 \pi \lambda^{2} \nu^{2} \Gamma\left(1-\frac{\rho}{2}\right)^{2} b_{h} \ln 2}{\mu_{\eta} \Gamma\left(\frac{1-\rho}{2}\right)^{2} n(n-1)}\right)\right) .
$$

Lemma 4 provides an analogous result to Lemma 3 for the sectorized pattern. Note that the result hold for fixed $\lambda$. We can easily perform a similar analysis to obtain scaling results in the directivity parameter, as summarized below.

Lemma 5: As $n \rightarrow \infty$, the entropy of a network of nodes with sectorized radiation patterns in $\mathcal{K}_{2}$ is bounded only if

$$
\lambda=\Omega(n) .
$$

The entropy bound will tend to a limit $h(n) \rightarrow b_{h}>0$ as $n \rightarrow \infty$ if

$$
\lambda(n)=c^{\frac{1}{2}} n
$$

where

$$
c=\frac{\eta \mu_{\eta} \Gamma\left(\frac{1-\rho}{2}\right)^{2} \ln r_{i}}{2 \pi r_{i}^{\eta} \nu^{2} \Gamma\left(1-\frac{\rho}{2}\right)^{2} b_{h} \ln 2} .
$$

Interestingly, we see from this result that directivity scaling is only dependent upon the prevailing path loss conditions (i.e., the exponent $\eta$ ) through the prefactor $c$. This is in contrast to the 
scaling law of Lemma 4, where dependence was exponential. With a little effort, we can deduce that in order to control network complexity in the large connection range regime, we would either need to scale the transmit power of each node like $O\left(n^{2} \ln n\right)$ or instead scale the directivity factor $\lambda$ like $O(n)$. This is the first concrete (and rather curious) conclusion that highlights the importance of directivity in random networks.

\section{CONCLUSIONS}

In this paper, we studied wireless network complexity by modeling a network as an RGG where each node has a random antenna orientation as well as a random position. We analyzed small and large typical isotropic connection range regimes for two different antenna radiation patterns and presented analytic scaling results that shed light on how network complexity can be controlled as the number of nodes in the network grows large. These results quantified scaling laws in the connection range, which is inherently linked to system parameters such as transmit power and frequency, as well as the directivity of transmissions. Although this work was largely from a theoretical perspective, it hints at an interesting juxtaposition of complexity and connectivity, since controlling the former may adversely affect the latter. It is the authors' hope that this work will inspire others to contribute to this developing field in wireless network research.

\section{ACKNOWLEDGMENT}

A. Cika and J. P. Coon wish to acknowledge the support of Moogsoft and EPSRC under grant number EP/N002350/1 ("Spatially Embedded Networks"). S. Kim acknowledges the support of the Samsung Research Funding \& Incubation Center of Samsung Electronics under Project Number SRFC-IT-160109.

\section{REFERENCES}

[1] G. Simonyi, "Graph entropy: A survey," Combinatorial Optimization, vol. 20, pp. 399-441, 1995 .

[2] J. Park and M. E. Newman, "Statistical mechanics of networks," Physical Review E, vol. 70, no. 6, p. 066117, 2004.

[3] M. Dehmer and A. Mowshowitz, "A history of graph entropy measures," Information Sciences, vol. 181, no. 1, pp. 57-78, 2011.
[4] R. Timo, K. Blackmore, and L. Hanlen, "On entropy measures for dynamic network topologies: Limits to manet," in Communications Theory Workshop, 2005. Proceedings. 6th Australian, pp. 95-101, IEEE, 2005.

[5] J.-L. Lu, F. Valois, M. Dohler, and D. Barthel, "Quantifying organization by means of entropy," IEEE Communications Letters, vol. 12, no. 3, 2008.

[6] J. Coon, "Topological uncertainty in wireless networks," in Global Communications Conference (GLOBECOM), IEEE 2016., 2016.

[7] J. Coon and P. Smith, "Topological entropy in wireless networks subject to composite fading," in International Communications Conference (ICC), IEEE 2017., 2017.

[8] T. Srinidhi, G. Sridhar, and V. Sridhar, "Topology management in ad hoc mobile wireless networks," in Proceedings of Real-Time Systems Symposium, Work-in-Progress Session, 2003.

[9] L. Pelusi, A. Passarella, and M. Conti, "Opportunistic networking: data forwarding in disconnected mobile ad hoc networks," IEEE Communications Magazine, vol. 44, no. 11, 2006.

[10] T. Kathiravelu, N. Ranasinghe, and A. Pears, "Towards designing a routing protocol for opportunistic networks," in Advances in ICT for Emerging Regions (ICTer), 2010 International Conference on, pp. 5661, IEEE, 2010.

[11] S. K. Sarkar, T. Basavaraju, and C. Puttamadappa, Ad hoc mobile wireless networks: principles, protocols and applications. CRC Press, 2007.

[12] T. S. Rappaport, S. Sun, R. Mayzus, H. Zhao, Y. Azar, K. Wang, G. N. Wong, J. K. Schulz, M. Samimi, and F. Gutierrez, "Millimeter wave mobile communications for 5G cellular: It will work!," IEEE access, vol. 1, pp. 335-349, 2013.

[13] M. D. Penrose et al., "Connectivity of soft random geometric graphs," The Annals of Applied Probability, vol. 26, no. 2, pp. 986-1028, 2016.

[14] M. Penrose, Random geometric graphs. No. 5, Oxford University Press, 2003.

[15] C. A. Balanis, Antenna theory: analysis and design. John Wiley \& Sons, 2016.

[16] G. Bianconi, "Entropy of network ensembles," Physical Review E, vol. 79, no. 3, p. 036114, 2009.

[17] G. Bianconi, A. C. Coolen, and C. J. P. Vicente, "Entropies of complex networks with hierarchically constrained topologies," Physical Review E, vol. 78, no. 1, p. 016114, 2008.

[18] K. Anand and G. Bianconi, "Entropy measures for networks: Toward an information theory of complex topologies," Physical Review E, vol. 80, no. 4, p. 045102, 2009.

[19] K. Anand, G. Bianconi, and S. Severini, "Shannon and Von Neumann entropy of random networks with heterogeneous expected degree," Physical Review E, vol. 83, no. 3, p. 036109, 2011.

[20] S. N. Chiu, D. Stoyan, W. S. Kendall, and J. Mecke, Stochastic geometry and its applications. John Wiley \& Sons, 2013.

[21] A. Goldsmith, Wireless Communications. Cambridge university press, 2005. 